\title{
Notulæ ad Plantas Japoniæ et Koreæ XXI
}

\author{
auctore
}

Takenoshin Nakai, Rigakuhakushi

447) Cephalotaxus nana, NaKar sp. nov.

Frutex 1-2 metralis. Rhizoma longe repens exquo innovationes hic illuc evolvent rubescenti-fuscum. Caulis ascendens vel erectus usque 6-7 ennis tum emortus et innovationes e basi agit. Cortex rubescentifuscus longitudine irregulariter fissus. Ramus verticillatus et foliis distichis, 1-2 ennis viridis. Squamæ gemmarum persistentes imbricatæ basi ramorum suffulta. Folia in situ spiralia sed disticho-patentia sessilia basi obtusa vel mucronata $1-4 \mathrm{~cm}$. longa supra viridia, costis elevatis, secus costas sulcato-impressa apice cuspidata, infra costis elevatis viridibus et inter costas et margines fasciis stomatum albolineata. Amenta mascula juvenilia axillaris cernua, evolutam non vidi. Amenta fominea in apice ramorum evoluta. Pedunculi subangulati. Squamæ 6 v. 8 decussatæ virides. Ovula in squama 2 sessilia ovoidea orthotropa. Fructus in quovis pedunculo 1-4 subglobosi basi obtusi apice subplani primo virides tum albescens deinde rubeseentes drupacei edules. Semen sphærico-ovoideum $13 \mathrm{~mm}$. longum $10 \mathrm{~mm}$. latum, testa crustacea fusca apice bicostata. Albumen carnosum. Embryo teres. Cotyledones $1 \mathrm{~mm}$. longæ. Radicula supera.

Nom. Jap. Hai-inugaya.

$\mathrm{Hab}$.

Yeso:- Prov. Ishikari : Kamuikotan (Kingo Miyabe). Sapporo (Ryokichi Yatabe). Nopporo (Takenoshin Nakai).

Prov. Siribeshi : Takashima (Jinzô Matsumura).

var. astringens, NAKAI.

Drupacea testa inedulis astringens, maturitate fuscens.

Hab.

Hordo: in montibus Aidzu prov, Iwashiro (TAKENoshin NAKAI). 
This remarkable Cephalotaxus seems to be widely distributed over Hokkaido. It was hitherto commixed with Caphalotaxus drupacea and sometimes considered to be a dwarf northern form of it. I have been in Hokkaido this summer. Going to Nopporo-forest I was astonished to find it making a pure bush under woods of Abies sachalinensis and Quercus crispula. I traced the root and found that the bush which covers certain area consists of a few stocks. Not only it branches from the base of stem, but its rhizome-like roots stretch under the ground, from which many aerial shoots come out. We tried to get young plants came out of seeds and those shoots often puzzled us. The shoots fruit when they become three feet high. Mr. MaEkawa, the assistant professor of Hokkaido University who went there with me, told me that the ripen fruits are reddish and edible. We tried several half-matured ones and they taste sweet however rich of regin. We kept a number of them and they became soon red. The Aidzu-one has the inedible fruits, but the habit is quite the same with Yeso-one. Both in Yeso and Aidzu this plant attains the same height with Sasa paniculata and often make social bushes.

448) Torreya nucifera, (Linné) Siebold et Zuccarini in Abh. Akad. Muench, IV. part 3 (1846) p. 232.

var. radicans, NAKAI.

Caulis e basi caespitoso-ramosus et fere 8 pedalis attingens, radicante-ascendens. Rami biennes claro-rubescentes. Truncus diametro vulgo quam $6 \mathrm{~cm}$. brevior.

Nom. Jap. Chabo-gaya.

Hab.

Hondo: in montibus Aidzu prov. Iwashiro (Takenoshin NakaI).

This Torreya grows on the mountains near Aidzu among broadleaved trees and Sasa paniculata. I could not find any big trees. Many wood-cutters whom I met with told me that in that locality Torreya is always shrubby and branches from root.

var. typica, NAKAI.

Arbor erecta vulgo magna.

Nom. Jap. Kaya-no-ki.

Hab.

Quelpært: in silvis Nokatji (TAQUET n. 1448), in silvis (TAQUET n. 6009). in silvis Hallasan $900 \mathrm{~m}$. (TAKenoshin NakaI n. 7717). Chodori (Tsutomu Ishidoya n. 279).

Corea: in silvis montis Pai-yang-san (Takenoshin NakaI $n$. 1015), in silvis insulæ Wangtō ('TAKENoshin NAKaI n. 7716).

Distr. Kiusiu, Shikoku et Hondo. 
In monte Paiyansan v. Paikamsan in silvis sparsim crescentes sunt vestustæ, fere $10 \mathrm{~m}$. altæ, truncis diametro 2 pedalis. Quum silva illa templi Yaik-un-san sit quod ante 1000 annos pede illius montis conditum, hæ plantæ nostris dubiæ sunt sive e plantatis elapsæ seu sint sane spontaneæ.

In Shodori lateris boreali-orientalis insulæ Quelpaert olim multum plantata sunt et nunc silvam magnificam faciunt. Truncus diametro 2 pedalis attingit annulos supra 200 enumerare possumus. Lignum tabulam laterunculariam facere optimum est, ita non paucæ cæsæ sunt, sed nunc officiale protectæ fere 5000 plantæ ibi manent. In Quelpaert attamen non rarum sed sparsim in silvis nascit. Dum ego et Prof. E. H. WILson in auctumno anni 1717 insulam perlustraverunt duas plantas sine dubio spontaneas inveniunt. Alia cum trunco supra $10 \mathrm{~cm}$. lato in rupibus secus cascade nascit aliaque in silvis nascit fere $4 \mathrm{~m}$. alta, satis vestustæ erant.

449) Picea koraiensis, NAKaI sp. nov.

P. ovobata, (non Ledebour) Komarov Fl. Mansh. I. (1900) p. 195 saltem p.p. NakaI Fl. Kor. II. (1911) p. 380 et Florula M't Paiktu-san (1918) p. 60 n. 23.

Arbor $16 \mathrm{~m}$. alta. Cortex rubescenti-fuscus. Rami declinato-ascendentes rubescenti-fusci glabri. Folia quadrangularia $0.9-1.3 \mathrm{~cm}$. longa apice acuta $1.5-2.2 \mathrm{~mm}$. lata leviter incurvata. Gemmæ rubescenti-fuscus ovatæ. Coni ovoideo-oblongi $7.5-8.3 \mathrm{~cm}$. longi $3.6-4.0 \mathrm{~cm}$. lati. Squamæ rigidæ lucidæ inferiores rotundatæ superiores obovatæ.

Nom. Jap. Chosen-harimomi.

Hab.

Corea sept.: districtu Musang (V. Komarov n. 82), in oppido Gōsui (Takenoshin Nakai n. 1880). in monte Atokryong (T. NaKaI).

Haec species $P$. Koyamæ simulat sed ramis pallidibus, foliis angustioribus et acerioribus, textura squamarum coni rigidioribus exqua distinguenda. In speciminibus exsiccatis cicatrices foliorum P. Koyamæ quam praesente distinctiores. Color ramuli quam Picea Koyamæ Piceae politæ simulat. Haec etiam in Manshuria versus Jalu latere dextro sane opposita districtu Heisanchin crcscit. Paludicola esse fere semper secus flumen invenitur, et non rarum societas cum Picea ajanensis et Abiete nephrolepide facit. Cum cortice rubescente insigna sit e satis distantia eam cognoscere possumus. Lignum est album atque molle nonnihil eidem Abietis firmæ simulat.

450) Pinus mukdensis, UeKI sp. nov.

P. Massoniana, (non Don) Komarov Fl. Mansh. I. (1900) p. 182.

Arbor $20 \mathrm{~m}$. non excedit. Rami robusti breves. Truncus arboris 
supra 200 annos diametro circiter $50 \mathrm{~cm}$. Cortex sordide v. atrofuscus plus minus cinerascens breve polygono-fissus. Cotyledones 6 (interdum 7-8) $2-5 \mathrm{~cm}$. longr. Hypocotyl fuscus rarius viridis $3 \mathrm{~cm}$. longus $1.5 \mathrm{~mm}$. latus. Folia plantæ juvenilis $2.5 \mathrm{~cm}$. longa serrulata. Rami pallide fuscentes. Folia vaginata cinerea basi fuscentia $8-10 \mathrm{~mm}$. longa apice membranacea. Folia genuina bina viridia acicularia 7.5$13 \mathrm{~cm}$. longa ad apicem pungentem serrulata lineis stomatosis dorso 6 ventre 5-6. Rami coniferi cinereo-fusci, foliis $8-16 \mathrm{~cm}$. longis serrulatis. Coni late ovoidei claro-fusci. Bracteæ apice pungentes.

Nom. Jap. Manshu-kuromatsu v. Hokuryo-matsu.

Hab.

Manshuria: Mukden (Homiki UEkI n. 2350).

Haec Pinus illustram silvam Borealis Mausolei Mukdensis format. Ante supra 200 annis ibi plantata sed nesciamus unde apportata erat. Aspectu Pino Thunbergii simulat sed foliis longioribus coni forma ct colore, cortice brevius fisso exqua distat.

451) Pinus funebris, Komarov Fl. Mansh. I. p. 177.

=Pinus densiflora, Siebold et Zuccarin! !

452) Pinus parviflora, Siebold et Zuccarini Fl. Jap. II. (1842) p. 27 t. 15.

Nom. Jap. Hime-komatsu.

Hab. Insula Ooryongto v. Dagelet Island. ('Tsutomu Ishidoya n. 2. TAKENOSHin NAKaI n. 4732. 4109).

Distr. Tsusima, Shikoku, Hondo et Yeso.

Ante 30 annos haec planta elementam gravem silvaticam facit, sed populi Coreani nimium multo caeserunt ut nunc paucæ in rupibus promontorii maneant. Maxima in Koriken vidi $30 \mathrm{~m}$. alta et ejus truncus diametro $1 \mathrm{~m}$. superat.

453) Thuja koraiensis, NAKAI nom. nov.

T. odorata, (non Marshall) Dor nom. nud.

T. Standishii, (non Carrière) NakaI Fl. Kor. II. (1911) p. 382.

T. japonica, (non Maximowicz) Komarov Fl. Mansh. I. p. 206. NAKA1 in Tokyo Bot. Mag. XXII. p. 53. et Report of Veget. M't Waigalbon in Chosen-iho extra edit. (1916) p. 66.

T. kongoensis, Dor in litt. NAkaI Veget. Diamond Mountains (1918) p. 163 n. 10 nom. nud.

Vulgo prostrata sed maxima erecta et $4 \mathrm{~m}$. alta. Truncus diametro usque $7-8 \mathrm{~cm}$. Cortex longitudine fissus rubescenti-fuscus. Rami radicantes vulgo elongati. Folia supra viridia vel viridissima interdum glaucescentia infra glauca squamosa, ramorum elongatorum acuta, brevium obtusa vel obtusiuscula. Coni stipes foliis squamosis 2-12 
ita $2-5 \mathrm{~mm}$. longus. Coni squamæ 4 pares, primarii squamosi late ovati recurvi v. late elliptici usque $4 \mathrm{~mm}$. longi, secundarii ovati vel rotundato-ovati $5-7 \mathrm{~mm}$. longi, tertii anguste obovati apice leviter recurvi, quartani lineares $v$. oblanceolati apice crassi. Coni ut toti ovoidei v. oblongo-ovoidei rubescenti-fusci subglaucescentes.

Nom. Jap. Nioi-nezuko.

Hab.

Corea: in montibus Rorinsan versus australem usque Kongosan, in monte Kongosan (Seiroku Honda, Tomijiro Uchiyama, Takenoshin NakaI n. 5080-5084). Koseimen (Takenoshin $N_{\text {akaI }}$ n. 1884). in monte Waigalbon (Takenoshin NakaI). Districtu Samsu (V. Kомarov n. 85). in monte Trunbon (Masatomi Furumi).

Haec Thuja est in Corea endemica et solum secus montes mediani continui continent Gokasan 2000 m., Trunbon 2000 m., Kisaiho 2100 m., Roho 2260 m., Rorinsan $2300 \mathrm{~m}$. usque ad Kongosan $1600 \mathrm{~m}$., sed copiosissime crescit. Boreale extremum incipit e districtu Samsu et terminat in extremo australe Kongosan. Virgultum densissimum facit ut nemo permeare possit, crescitque perinde inferiore quam Pinus pumila, tum per utræque interjectionem sensim virgultum posterioris transit. Folia fricta odorem Thujæ occidentalis agunt.

454) Tsuga Sieboldii, Carrière Traite Conif. (1855) p. 186.

Nom. Jap. Tsuga.

Hab.

In insula Ooryongto v. Dagelet Island, in montibus copiosa. (TAKenoshin Nakai n. 4110, Tśtomu Ishidoya n. 3).

Arbor magna in hac insula maxima truncus diametro 4 pedalis attingit. Olim multæ fuisse dicitur. Nunc tamen in silvis montium Koriken non rara.

455) Populus jesoensis, Nakai sp. nov. (Sect. Leuce).

Arbor circ. 7 metralis ramosus. Truncus teres. Cortex sordide fusco-cinerascentes. Gemmæ ovatæ reginoso-viscidæ. Petioli 20-65 mm. longi apice laterali compressi virides basi incrassati. Lamina glaberrima late ovata vel subdeltoidea apice acuta $v$. acuminata basi truncata, minora late cuneata et non glandulifera $37-90 \mathrm{~mm}$. longa $32-78 \mathrm{~mm}$. lata margine obtuse crenulato- $v$. incurvato-serrata supra viridissima lucida stomatibus destituta, infra viridia. Amentam non vidi.

Nom. Jap. Ezo-yamanarashi.

Hab.

Yeso: Prov. Teshio: in silvis Kami-nayoro (Takenoshin NakaI). Prov. Kitami : Abashiri (S. Sugryama).

This Populus is an allied species of Populus Sieboldii, from which 
it may be readily distinguishable in having glabrous leaves and lacking of glands at the base of laminæ. The smallest leaves alike to those of Populus tremula var. Davidiana, but the branchlets and petiols are stouter and always green. It grows on dry soil among the woods consisting of Alnus borealis, Alnus tinctoria, Salix caprea, Quercus crispula, Betula japonica, Ulmus laciniata, Tilia japonica, Maackia amurensis var. Buergerii and Juglans Sieboldiana etc. Populus tremula var. Davidiana which is a commonest tree in North-Corea grows in Hokkaido too. We have in our University Herbarium two specimens collected at Moiwa Hill and the foot of mount Tokachidake.

456) Asarum Sieboldii, Miquel Prol. Fl. Jap. (1866-7) p. 66.

var. seoulensis, NAKaI in Fedde Repert. (1914) p. 267.

A. Sieboldii, Maximowicz in Mél. Biol. VIII. (1871) p. 397 pp. NakaI Fl. Kor. II. 175 pp.

Nom. Jap. Ke-usuba-saishin.

Hab. Manshuria austro-orient. ad Ussuri superiorem. Maio 1860 (Maximowicz). Specimen in Herbario Bogoriense servatum.

Nova in Flora Manshurica.

457) Cercidiphyllum japonicum, Siebold et ZuCCARINI in Abhandl. Akad. Muench IV. part 3, p. 238 (1846).

var. magnificum, NAKAI.

A typo sequenti modo dignoscendum.

Typicum.

Cortex rami diametro circ. 3-5 cm. jam longitudine fissus. Ramuli graciles. Petioli graciles vulgo rubescentes $1-1.5 \mathrm{~mm}$. crassi. Lamina maxima $8-9 \mathrm{~cm}$. longa et lata supra fere plana infra glauca venis moderate elevatis.

Magnificum.

Cortex rami quam $20 \mathrm{~cm}$. latioris longitudine findere incipit, ita truncorum quam typicus multo brevius fissus semper cinereus. Ramuli robusti. Petioli robusti virides $1.5-3 \mathrm{~mm}$. crassi. Lamina maxima 13-14 $\mathrm{cm}$. longa et lata supra distincte rugulosa infra glauca venis eximie elevatis.

Nom. Jap. Uchiwa-katsura.

Hab.

Hondo: in monte Shirane et Konsei, Nikko ('Takenoshin NakaI).

This Cercidiphyllum is evidently different from $C$. japonicum in having larger leaves and ashy bark which does not split till it becomes at least 20 years old. On the feet of cited mountains where Cercidiphyllum japonicum is growing, seldom comes to grow aside, then the characteristics become more conspicuous to our eyes. In June I have 
collected its flowers and in September the fruits, but they never differ from the type.

458) Pyrus aromatica, Kikuchi et NakaI in Tokyo Bot. Mag. XXXII. (1818) p. 32.

$P$. nambuana,. Kordzumi in Tokyo Bot. Mag. XXXIII. (1919) p. 128.

P. jucunda, KorDzumi l.c.

Pyrus nambuana is an individual of Pyrus aromatica having smaller fruits. The leaves become often roundish in our type and the mode of serrations concords perfectly to ours. Dr. Kordzumi says 'foliis ab initio fere glabris', but in his type specimen having adult leaves has hairs sparingly on the pedicells and the costx, by which the pubescence of the younger plants may be imagined. Pyrus jucunda has hairs too along the base of costæ even in its adult stage.

var. obovoidea, (KoIdzumi) NakaI.

P. obovoidea, Kordzumi in Tokyo Bot. Mag. XXXIII. (1919) p. 123.

Fructus obovoidea. Cetera ut typo.

Nom. Jap. Ko-yahadzunashi.

Hab.

Hondo: in oppido Ayaori prov. Rikuchu.

forma wayamana, (KoIDzumi) NAKAI.

Pyrus wayamana, Koidzumi l.c.

Forma cum fructibus majoribus, calyce deciduo.

forma iwatensis, (KoIdzumi) NaKaI.

P. iwatensis, Kordzumi 1.c. p. 127.

forma hortensis cum fructibus majoribus.

Nom. Jap. Yahadzu-nashi.

459) Pyrus hondoensis, NakaI et Kikuchi in Tokyo Bot. Mag. XXXII (1918) p. 32.

var. microphylla, NaKaI et KIKUCHI.

Rami breves apice spinescentes. Folia parva 1-3 cm. longa.

Nom. Jap. Ko-aonashi.

Hab.

Hondo: in Ipponmatsu pede montis Fuji (Aкıо KıкUCHI).

var. lasiogyna, NakaI et Kikuchi.

Styli basi pilosi. Cetera ut typo.

Hab.

Hondo: in monte Handayama (牛田山) oppidi Soehimura (傍陽村) districtu Chiisagata (小縣郡) prov. Shinano (KANAE YAMAGISHI).

Pyrus amoena, Koidzumi 1c. p. 124 and Pyrus incubacea, Koldzumi 
1.c. are Pyrus serotina. The former has leaves rounded at their base and the latter cordate.

460) Pyrus insueta, Koldzum in Tokyo Bot. Mag. XXXIII. (1919) p. 124.

forma longe-pedunculata, (KoIDzumi) NakaI.

$P$. longe-pedunculata, Kordzumi 1.c. p. 126.

forma hortensis cum pedunculo longiore et fructu majore.

Pyrus insueta is near to $P$. aromatica but differs from that by its almost crenate serrations. Calyx is persistent or deciduous.

461) Pyrus Kikuchii, NaKaI sp. nov.

Cortex trunci pallidus lenticellis subnullis. Rami biennes virides vel fusco-virides glabri lenticellis rotundatis vel oblongis sparsim punctulati, hornotini rufo-pilosi. Petioli usque $4.5 \mathrm{~cm}$. longi primo rufopilosi demum glabrescentes. Stipulæ caducæ filiformes. Lamina foliorum primo supra rufo-pilosula costis dense rufo-pilosis, margine et infra densissime rufo-pubescentes demum fere glabrescentes virides, ovata basi leviter cordata, apice acuminatissima margine setoso-serrata usque $9 \mathrm{~cm}$. longa $7 \mathrm{~cm}$. lata. Pedicelli usque $4.5 \mathrm{~cm}$. longi, saltem infra medium rufo-tomentosi. Calyx ovatus glaber, limbo aperto lobis acuminatissimis glanduloso-serrulatis extus glabris, intus dense rufopubescentibus. Petala alba obovato-rotundata. Pomum globosum apice leviter excavum calyce persistente coronatum, basi excavum, facie primo viride demum fuscens lenticellis diametro 1-2 $\mathrm{mm}$. latis punctulatum, diametro $7-7.5 \mathrm{~cm}$., sapore duro ingrato, cellulis induratis copiosissimis. Ovarii loculi medio elongato-aperti. Semen nigrum oblique obovatum $12 \mathrm{~mm}$. longum. Pedunculi fructiferi medio $3 \mathrm{~mm}$. basi $8-9 \mathrm{~mm}$. crassi, virides lenticellis minutissime punctulati.

Nom. Jap. Kono-watashi 耕之渡.

In hortis Japonensis rarius culta.

I don't know whence this cultivated pear came from. Except the stout and elongated nearly trailing branches and the rusty hairs on the leaves, branches, flower-stalks and inside of calyx this is nearest to Pyrus serotina. I have named in honour of $\mathrm{Mr}$. Akio Kikuchi, the director of the Kanagawa Agricultural Experimental Station and the author of Pyrtis hondoensis, $P$. aromatica and $P$. crassipes who are specially investigating on Pears.

462) Pyrus lasiogyna, KoIdzumi in Tokyo Bot. Mag. XXXIII (1919) p. 125.

var. lanuginosa, NAKAI.

Ramus et folia juvenilia albo-lanata. Calyx extus pedunculique albo-lanuginosa. Styli basi pilosi. Fructus rotundatus utrinque ex- 
cavus extus viridis, sapore gratissimo eximie succoso. Pcdunculi incrassati.

Nom. Jap. Nijisseiki 廿世紀.

This is the best Japanese Pear hitherto known. Though its fruit is difficult to preserve on account of the richness of juice, it is the nicest and has best flavour. In every respect it surpass the European pears. It is said to have been raised from two parents which are kept only by its raiser, yet we can point that at least one of its parents is Pyrus lasiogyna. In crossing the present variety with other pears the pubescence is always dominant.

463) Pyrus rufo-ferruginea, Kordzumi in Tokyo Bot. Mag. XXIX. (1915) p. 311.

var. tremulans, (KoIDzumi) NAKaI.

P. tremulans, KoIdzumi 1.c. XXXIII. (1919) p. 126.

P. insulsa, KoIDzumi 1.c. p. 127.

$P$. tremulans and $P$. insulsa are one and the same variety of Pyrus rufo-ferruginea, differing merely in the small serrations and more glabrous leaves.

Hab. pede montis Hayachine prov. Rikuchu, Hondo.

464) Albizzia coreana, NAKAI.

A. Lebbek (non Willdenow) NakaI Tokyo Bot. Mag. XXVII. (1913) p. 131.

Arborea 6-8 metralis. Ramus rubescens glaber lenticellis fuscis punctulatus. Petioli $3-5.5 \mathrm{~cm}$. longi sparsim piloso, glandula unica media posita (rarius circa basin v. apicem). Folia pari-bipinnata. Pinnæ foliolis 5-10 paribus. Rachis pinnæ pilosa. Foliola supra costa angusta basi attenuata infra costa basi acuta $v$. subtruncata, apice obtusa v. mucronato-apiculata, supra viridia sub lente sparsim pilosa demum glabra, infra pallida pilosula, margine integerrima pilosa sessilia 1.7-4.5 cm. longa 5-20 mm. lata. Flores capitati subsessiles. Pedunculi axillares in quaque axillis $1-3$ verticale positi $6-6.5 \mathrm{~cm}$. longi pilosi. Pedicelli $1 \mathrm{~mm}$. longi pilosi. Calyx ovatus pilosus 3 mm. longus 5 dentatus. Corolla 5-6 mm. longa pilosa lobis late lanceolatis apice pubescentibus. Stamina 30-40, $25 \mathrm{~mm}$. longa lilacina. Legumen pendulinum breviter stipitatum $8-17 \mathrm{~cm}$. longum, $2 \mathrm{~cm}$. latum lucidum fuscum. Semina oblongum compressum $7 \mathrm{~mm}$. longum 4-5 mm. latum.

Nom. Jap. Ohba-nemu.

Hab. Corea, in montibus Mokpo (T. Uchiyama, T. Nakai).

465) Cassia occidentalis, Linne Sp. Pl. I. (1753) p. 337. Edwards Bot. Regist. I. (1815) t. 83. De Candolle Prodr. II. (1825) p. 497. 
Forbes et Hemsley in Journ. Linn. Soc. XXIII. (1887) p. 211. Trimen Handb. Fl. Ceyl. II. (1894) p. 105. Matsumura in Tent. Fl. Lutch. in Journ. Sci. Coll. Imp. Univ. Tokyo XII. (1899) p. 438. Matsumura et Hayata Enum. Pl. Form. p. 115. Hayata Icones Pl. Form. I. (1911) p. 210 .

Nom. Jap. Kusa-senna.

Hab.

Formosa: Tansui (Tomitaro Makino). Koshun (Chutaro O. watari). Reiga-ryo(Chutaro Owatari). Koshun (Chutaro Owatari) Burokei, Shinchiku et Tainan (?)

Bonin : (Ryōkichi Yatabe, Shigeki Nishimura).

466) Cassia torosa, Cavanilles Descripcion Plant. (1801) p. 131. Persoon Syn. Pl. I. (1805) p. 457. De Candolle Prodr. II. (1825) p. 491.

C. chinensis, (non LiNné) JacQuin Icon. Pl. rariorum t. 73 (1781). Persoon 1.c.

C. Sophera, (non Linné) Forbes et Hemsley in Journ. Linn. Soc. XXIII. (1887) p. 211 p.p.

C. occidentalis, (non Linné) Matsumura in Tokyo Bot. Mag. XVI. (1902) p. 100 et Ind. Pl. Jap. II. 2. (1912) p. 253. Makino in Innuma's Somokudzusetsu ed. rev. II. (1910) p. 607. Pl. 472.

Nom. Jap. Habusō.

In Japonia, Liukiu et Formosa culta olim e China introducta. 467) Lespedeza Oldhami, Mrguel Prol. Fl. Jap. p. 236.

L. Buergeri, Mrquel var. Oldhami, Maximowicz in Acta. Hort. Petrop. II. p. 354. Palibin Consp. Fl. Kor. I. p. 65. Nakai Fl. Kor. I. p. 155.

L. Buergeri, (non Miquel) Palibin 1.c. Nakai 1.c.

L. Buergeri var. præcox, NAKAI in Tokyo Bot. Mag. XXV. (1911) p. 55. Veg. Isl. Wangtô (1914) p. 9. Fl. Kor. II. p. 467.

L. præcox, NakaI in Cat. Sem. et Spor. Hort. Bot. Univ. Imp. Tokyo (1914) p. 20 1. 566. et Veg. Mt. Chirisan (1915) p. 36 et 84, Veg. Diamond mountains (1918) p. 176 n. 379 a.

L. Friebeana, Schindler in Fedde Repert. (1911) p. 514. Craib in P1. Wils. IV. p. 111.

L. bicolor var. intermedia (non Maximowicz) Palibin 1.c. p. 64, saltem pro parte.

Nom. Jap. Chôsen-kihagi.

Nom. Cor. Amun-pisarite, Nam-pisari, Pisari.

Hab. Kiusiu et Corea.

Kiusiu: Nagasaki (in Herb. Buitenzorg). 
Corea: Chirisan (Takenoshin Nakai n. 46, 74, 86, 118). inter Seiyu et Chôjô (T. Nakai n. 1196). Namsan (Tomjiro Uchiyama, Faurie n. 412) insula Okto (T. NakaI n. 814), prope Tap Tong (Sontag) Koang Nyong (Tamezo Mori n. 288.). Suigen (Homiki Ueki n. 649). Neihen (HanjiRō Imai n. 120), in monte Manjyokusan ( $T$. NakaI) in montibus Ouensan (Faurie n. 51. 411). Hekidô (T. NakaI n. 1981) Shôjô (T. Nakai n. 1980).

var. tomentella, NakaI Veg. Diamond Mountains (1918) p. 176 n. $379 \mathrm{~b}$.

L. Friebeana, NA KaI Veg. Isl. Wangtô (1914) p. 9.

Folia supra sericea infra sericeo-tomentosa. Pedunculi et calyx sericei.

Nom. Jap. Shimofuri-kihagi.

Hab. Corea.

Mokpo ('Tsutomu Ishidoya n. 16). Kongosan (T. NakaI n. 55675571), insula Wangto (T. NAKaI n. 592).

468) Phaseolus calcaratus, Roxburgh Hort. Beng. p. 54. Fl. Ind. III. p. 289. Baker in Hook. Fl. Brit. Ind. II. p. 203.

$P$. radiatus $\gamma$. subtrilobata, Franchet et Savatier Enum. Pl. Jap. I. p. 111. Kanime.

Nom. Jap. Tsuru-azuki, Kome-azuki, Shabon-mame, Nirako,

Icon. Somokudzusetsu Vol. 13 f. 30. Honzozufu Vol. 43. f. $12 \cdot$ In agris colitur.

469) Phaseolus chrysanthos, Savi in Nuov. Giorn. Sc. III. (1822) p. 318 et in Mem. Soc. Torino XXXVIII. (1835) p. 177.

$P$. radiatus var. aurea, (non Prain) Matsumura in Tokyo Bot.

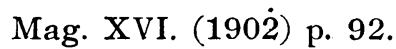

Planta erecta $\nabla$. bene evoluta apice flexuosa rarius subvolubilis. Legumen glabrum $\mathbf{v}$. hispidum, maturitate nigrum $\mathbf{v}$. flavescens semper pendulum. Semina fere sphærica saepe subangulata, eicatrice alba.

Nom. Jap. Azuki.

In agris colitur.

Formæ multæ adsunt e.g.

Natsu-azuki v. Shonagon.

Semina sordide rubra usque $4-5 \mathrm{~mm}$. longa.

Aki-azuki v. Chunagon.

Semina sordide rubra usque 5-6 $\mathrm{mm}$. longa.

Oh-azuki v. Dainagon.

Semina sordide rubra 6-7 $\mathrm{mm}$. longa.

Uzura-azuki. 
Semina sordide rubra irregulariter nigro-punctata.

Ao-uzura.

Semina viridia irregulariter nigro-punctata.

Shiro-azuki.

Semina sordide alba 5-6 mm. longa.

Cha-azuki.

Semina fuscescentia.

Nonko-azuki.

Semina alba sed circa cicatricc atra.

Kuro-azuki.

Semina nigra ut Phaseolus mas.

etc. etc.

Olim omnes e China introducta.

470) Phaseolus minimus, Roxburgh Fl. Ind. III. p. 290.

P. trilobus, (non Arton) Matsumura Tokyo Bot. Mag. XVI. (1902) p. 92.

Nom. Jap. Yabu-tsuru-azuki.

Hab. in incultis Japoniæ.

471) Viola arcuata, Blume Bijdragen I. (1825) p. 58. Miquel Fl.

Ind. Bat. I. (1859) p. 114.

$V$. distans, (non Wallich) Backer Schoolflora I. (1911) p. 66.

V. japonica, (non Langsdorf) Miquel Cat. p. 9 apud Maximowicz. Mél. Biol. IX. p. 750.

V. palustris, (non Linné) Thunberg Fl. Jap. (1784) p. 326.

V. verecunda, Asa Gray Bot. Jap. in Mem. American Acad. Arts and Science Vol. VI. new series (1859) p. 382. Miquel Ann. Mus. Bot. Lugd. Bat. II. p. 155. BAKER and Moore in Journ. Linn. Soc. XVII. 379. Maximowicz Mél. Biol. IX. p. 750 excl. var. Henry List. Pl. Form. p. 18. Miyabe Fl. Kuril. p. 219. Palibin Consp. Fl. Kor. I. p. 36. Diels in Engler Bot. Jahrb. XXIX. p. 477. Borssieu in Bull. Herb. Boiss. (1900) p. 1075. Komaroy Fl. Mansh. III. p. 71. Matsumura et Hayata Enum. Pl. Jap. p. 31. Nakai Fl. Kor. I. p. 65. II. p. 445 et in Tokyo Bot. Mag. XXX (1916) p. 279.

Nom. Jap. Tsubo-sumire.

Hab. Manshuria, Corea, Quelpaert, Sachalin, Kuril, Yeso, Hondo, Shikoku, Kiusiu, Formosa, China et Java.

Sachalin : sine loco speciali (G. NAKahara).

Yeso: Asahigawa (Hineo Komzumi). Sapporo (Y. Tokubuchi, Kingo Miyabe). Sarurusandô (Kingo Miyabe). Hakodate (Jinzo Matsumura).

Hondo: monte Hakkodasan prov. Mutsu (Hideo KoIDzumi), monte 
Iwatesan prov. Rikuchu (GenjI NAKAHARA), monte Gassan prov. Uzen (Issei Tamaki), monte Hondojisan prov. Uzen (Ryokichi Yatabe), monte Rokujiurigoe prov. Uzen (Saburo OkUBo), monte lidesan prov. Uzen (Genji Nakahara). Aidzu prov. Iwashiro (Genji Nakahara), in monte Azumasan prov. Iwashiro (Genji Nakahara). Fukushima prov. Iwashiro (Genji Nakahara), in monte Asakusayama prov. Echigo (Masao Nakamura), in monte Shirouma prov. Shinano (Shinsuke Kodama). Mito prov. Hitachi (Isaku Ando). Nikko prov. Shimotsuke (Komajiro Sawada, Jinzo Matsumura). Tokyo (Jinzo Matsumura). Oji prov. Musashi (Jinzo Matsumura). Shimura prov. Musashi (TakeNoshin Narai), in monte Ohyama prov. Sagami (Sadahisa Matsuda), in monte Amagisan prov. Izu (Jinzo Matsumura). Hatchogaike prov. Izu (Jinzo Matsumura), ad ripas fluminis Abe prov. Suruga (Takenoshin NakaI), in monte Hakusan prov. Kaga (Jinzo Matsumura). Yamato (T. TADA). Kawarabayashi prov. Tamba (KeI TAKenouchi). Yunogo prov. Mimasaka (Genichi KoIDzumi), in monte Hôbenzan prov. Suwo ('T. Goya). Hikami prov. Suwo (Jiuro Nikai n. 377).

Shikoku : oppido Kamomyomura prov. Awa (Jiuro Nikai n. 2483). oppido Nanogawamura prov. Tosa (Tomitaro Makino).

Insula Tsushima: Izuhara (K. Hrrata).

Kiusiu: Kashundake prov. Buzen (HAmada). Shiroyama prov. Satsuma (Jinzo Matsumura). Kiire prov. Satsuma (Shozo Yajima). Kagoshima prov. Satsuma (Shozo YajıMa).

Insula Dagelet: Koriken (TAKenoshin NakaI n. 4444. 4598). Ohbokdong (TAKENoshin Nakai n. 4445).

Manshuria: circa stationem Radde prov. Amur. (V. Komarov n. 1116).

Corea: Shingishu (Masatomi Furumi n. 42). Kangei (Mills n. 386). Pyengyang (Hanjiro Imai). Seoul (Mirlls n. 842). Onseiri (Takenoshin Nakai n. 5658). Sea Kongô (Takenoshin Nakai $n$. 5657). Saihokumen (Takenoshin Nakai). Seoul (Tamezo Mori). Suigen (Homiki Ueki), in monte Namsan (Tamezo Mori, Tomijiro Uchiyama), in monte Kongosan (Tomijiro Uchiyama), in monte Paukhan (SoNTAG).

Quelpært: in monte Hallasan (Takenoshin NakaI). Hongno (TAQUET n. 2643, 595, 4576, 4585, 4588, 4595, 4596, 5398). Hiotien ('TAQuet n. 4576), in herbidis (TaQuet n. 2637).

Formosa : Taihoku (Takiya Kawakami n. 1672). Hichiseitonzan, Heichosho (T. Nagasawa).

Distr. China, Java, India et Ceylon.

472) Viola diamantiaca, NakaI sp. nov. 
V. serpens, (non Wallich) NaKaI Fl. Kor. I. p. 68 et Veg. Diamond M'ts (1918) p. 179.

Flores destituti. Planta Violæ Rossi accedit sed stolonifera.

Rhizoma crassum oblique repens vermiculare. Folia omnia radicalia, petiolis pilosis $8-21 \mathrm{~cm}$. longis apice pubescentibus varie purpureo-maculatis, laminis cordatis e basi sinus ad apicem $6.5-10 \mathrm{~cm}$. longis attenuatis $\mathbf{6 - 1 1} \mathrm{cm}$. latis supra viridibus pilosis infra pallidibus toto præcipue secus venas pubescentibus margine crenatis. Stolones elongati. Flores cleistogamosi foliis 3-5 plo breviores. Bracteæ 2 supra medium pedunculi positi. Calyx glaber. Fructus $13 \mathrm{~mm}$. longus purpureo-maculatus. Semina $2-2.5 \mathrm{~mm}$. lata.

Nom. Jap. Fuki-sumire.

Hab. Corea media: in montibus Kongosan (TomyIro UchIyama, Takenoshin Nakai n. 5661-2, 6067).

473) Viola multifida, (non Miller nec Willdenow) Makino in Tokyo Bot. Mag. XVI. (1902) p. 125. XIX. (1905) p. 144. XXVI. (1912) p. 157.

V. incisa var. multifida, Franchet et Savatier Enum. Pl. Jap. II. (1879) p. 284.

= Viola polysecta, NAKAI nom. nov.

474) Viola serpens, (non Wallich) Komarov Fl. Mansh. III. p. 62 quoad plantam e Corea = Viola Selkirki, Pursh. ! !

475) Edgeworthia towentosa, (Thunberg) NakaI comb. nov.

Magnolia tomentosa, Thunberg in Trans. Linn. Soc. II. (1794) p. 336 excl. Syn. Mag. glauca et Kobus Koempfer Icon. sel. tab. 42.

Edgeworthia chrysantha, Lindley Journ. Hort. Soc. I. p. 148. Bot. Regist. XXXII. (1849) t. 48. Matsumura Ind. Pl. Jap. II. 2. (1912) p. 388. Rehder et Wilson in Pl. Wilson VI. (1916) p. 550.

E. papyrifera, Siebold et Succarini Fl. Jap. Fam. Nat. II. (1846) p. 199. Mrquel. Prol. Fl. Jap. p. 299. Franchet et Sayatier Enum. P1. Jap. I. p. 405.

Nom. Jap. Mitsumata.

In montibus $\mathbf{v}$. in agris Japonensibus culta, olim e China introducta.

476) Elæagnus nagasakiana, NAKAI sp. nov.

Elæagni glabræ simulans sed exqua floribus $2-3$ plo majoribus dignoscenda.

Caules radicantes ? fusco-lepidoti teres. Rami teres leviter angulati fusco-lepidoti. Petioli 4-9 $\mathrm{mm}$. longi rubescenti-fusco-lepidoti. Folia late lanceolata $\mathrm{v}$. oblongo-lanceolata basi rotundata apice attenuata integerrima 3-6 cm. longa $1.7-3 \mathrm{~cm}$. lata. Flores axillari-gemini 
interdum subumbellati. Pedunculi brevissimi 1-3 mm. longi. Pedicelli $5 \mathrm{~mm}$. longi ad apicem sensim incrassati rubescenti-fusco-lepidoti. Ovarium fusiforme $6 \mathrm{~mm}$. longum. Sepala elongato-campanulata angulata $11 \mathrm{~mm}$. longa dense fusco-lepidota, lobis 6-7 mm. longis.

Nom. Jap. Hana-tsuru-gumi.

Hab. Kiusiu: Inasadake circa Nagasaki prov. Hizen. 10. IX. 1865 (Dr. E. Weiss n. 334).

Detexi hanc in Herbario Bogoriense.

477) Pieris elliptica, (Siebold et Zuccarini) Nakai comb. nov.

Andromeda elliptica, Siebold et Zuccarini Fl. Jap. Fam. Nat. II. (1846) p. 129.

A. ovalifolia, (non Wallich) Miquel Ann. Mus. Bot. Lugd. Bot. I. p. 30. Maximowicz in Mél. Biol. VIII. p. 620. Franchet et Savatier Enum. Pl. Jap. I. p. 285. Boissieu in Bull. Herb. Boiss. (1897) p. 913.

Pieris ovalifolia, (non G. Don) Makino in Tokyo Bot. Mag. VII. (1894) p. 213.

Nom. Jap. Nejiki v. Kashioshimi.

Hab.

Kiusiu: prov, Higo (?), Iwadake prov. Buzen (Jinzo Matsumura), Toyotsu prov. Buzen (HAMAda), Hyakkan-ishi prov. Higo (?).

Shikoku : oppido Niuta (入何) districtu Myōzai (名西) prov. Awa (Jiurō NikaI n. 2497), Hashikurayama prov. Awa (?).

Hondo: Nunobiki prov. Settsu (Jinzo Matsumura), Ōmineyama prov. Yamato (Jinzō Matsumura), oppido Ōnomura districtu Taga prov. Hitachi (Isaku Andō), Nikkō (Jinzo Matsumura, Komajirō SAWAdA), prov. Kawachi (T. TADA), monte Ontake prov. Shinano (Gen-ichi KoIdzumi), Fukushima prov. Iwashiro (Genji Nakahara), Hikami districtu Yoshiki prov. Suwo (JruRō NikaI n. 427). in insula Miyajima prov. Aki (Bunzō Hayata).

An endemic plant of Japan! It is a shrub not higher than 15 feet. The leaves and flowers are not downy. Corolla is white instead of pale flesh-colour.

478) Rhododendron scabrum, G. Don Gard. Dict. III. (1834) p. 846 excl. syn.

R. poukhanense, (non Lévell..é) Komatsu in Tokyo Bot. Mag. XXXII. (1918) Jap. p. 12 p.p.

$R$. poukhanense forma obtusifolium et $\mathrm{f}$. acutifolium, Komatsu 1.c. p. 37-38.

Folia ovata v. ovato-lanceolata scabra. Calycis lobi obtusi interdum acuti. Stamina 10 vel abortive 9-7 papillosa. Antheræ 
flavescentes.

This is an extreme variety of common Japanese hill-Rhododendron - Yamatsutsuji or Rhododendron Kaempferi. The ty pe of GeORGE Don is a form having broad leaves and ten stamens, but when it diminishes in the breadth of leaves and in the number of stamens, it passes gradually into Rhododendron Kæmpferi. There are no such variable Rhododen. dron like $R$. Kæmpferi in Japan. At any spots where $R$. Kæmpferi grows abundantly, we can gather any coloured flowers which varies among white, brick.red and purple. The variety mikawanum of MAKINO is therefore simply an individual of $R$. Kæmpferi having purple flowers. But sometimes special colour predominates in special places. In such a case one would incline to distinguish special-coloured one as a distinct variety. The garden form identified by S. Komatsu are as follows.

1. Mikawa-murasaki.

2. Edo-murasaki.

3. Hoso-mikawa-murasaki.

4. Tsuno-mikawa-murasaki.

5. Murasaki-zai.

6. Akebono-liukiu

7. Katsu-iro.

8. Waka-murasaki.

9. Gabisan.

10. Hatsushimo v. Akebono.

11. Nishikino-mori v. Nishikino-tsukasa.

12. Asukagawa-shibori.

13. Hōōden.

var. Kæmpferi, (Planchon) Nakai.

Rhododendron Kæmpferi, Pl.anchon Fl. de Serres IX. (1853) p. 79. Millais Rhod. (1918) p. 197.

R. Sieboldii, Miquel Ann. Mus. Bot. Lugd. Bat. I. p. 33.

Azalea indica, (non Linne) Thunberg Fl. Jap. p. 84.

Rhododendron indicum var. Kæmpferi, Maximowicz Rhod. Asiæ orient p. 38. Franchet et Savatier Enum. Pl. Jap. I. p. 291. MatsuMURA Ind. Pl. Jap. II. p. 460.

Folia ovata v. ovato-lanceolata v. obovato-lanceolata $\mathbf{v}$. oblonga. Stamina 5 interdum 6-8 papillosa v. fere glabra. Antheræ flavescentes.

Nom. Jap. Yama-tsutsuji.

This one may be distinguished into four remarkable forms, but the connective links are found everywhere.

\section{f. 1. purpureum, NakaI.}


Rhododendron Kæmpferi var. purpureum. NAKaI in sched.

$R$. purpureum, Komatsu in Tokyo Bot. Mag. XXXII. (1918) p: 16.

Folia late lanceolata v. lanceolato-oblonga scabra. Calycis lobi lanceolati. Stamina vulgo 5.

f. 2. latifolium, NAKaI.

Folia oblonga v. ovato-oblonga scabra. Calycis lobi obtusi. Stamina vulgo 5 .

f. 3. tubiflorum, NAKAI.

R. Kæmpferi var. tubiflorum, Komatsu 1.c. p. 17.

Folia ovato-lanceolata v. oblongo-lanceolata. Corolla angustior. Calycis lobi obtusi. Stamina vulgo 5 .

f. 4. angustifolium, NAKaI in sched. .Komatsu l.c.

Folia lineari-lanceolata. Calycis lobi obtusi. Stamina vulgo 5. For gardening or for genetics it necessitates to distinguish such varieties, but in the field the distinctions make no use at all.

479) Sideroxylon liukiuense, NAKAI sp. nov.

S. ferrugineum, (non Hooker et ARNold) Henry List. p. 58? MAtsumura et Hayata Enim. Pl. Form. p. 227. Matsumura Ind. Pl. Jap. II. 2. p. 484 p.p.

Differt a $S$. ferrugineo foliis obovatis saepe obtusis infra pallide fuscente $v$. argenteo-sericeis.

Rami primo ferrugineo-sericei sed mox glabrescentes. Folia obovata v. oblonga basi attenuata $v$. subcuneata apice obtusa $v$. acuta supra sparse pilosa infra argenteo $v$. subfuscente sericea, $2.5-10 \mathrm{~cm}$. longa $1.2-4.8 \mathrm{~cm}$. lata. Flores axillari-glomerati gemini patentes non vidi. Pedunculi ferrugineo-sericei. Sepala 5 late ovata v. rotundata quincuncialia rufo-pilosa. Drupa $1 \mathrm{~cm}$. longa oblonga pedunculo circ. 5 mm. longo.

Nom. Liuk. Jiiki.

$\mathrm{Hab}$.

Liukiu: Ohura insulæ Okinawa (Setsusaburo Tanaka), insula Okinawa (Jinzō Matsumura). Nase insulæ Oshima (Shözōo Yajima).

Formosa: Kelung (S. Nagasawa et C. OWatari).

480) Mimulus inflatus, (Mrquel) NaKai sp. nov.

Torenia? inflata, Mrquel Prol. Fl. Jap. (1867) p. 356. Franchet et Savatier Ènum. P1. Jap. I. p. 345. Maximowicz in Mél. Biol. IX. (1876) p. 659.

Mimulus nepalensis forma japonica, Mrquel Prol. Fl. Jap. (1866) p. 48 .

M. nepalensis $\beta$. japonicus, Maximowicz in Mél. Biol. IX. (1874) p. 401. Franchet et Savatier Enum. Pl. Jap. II. (1879) p. 455. 
M. nepalensis, (non Bentham) Franchet et Savatier Enum. Pl. Jap. I. (1875) p. 343.

M. nepalensis var. japonica, Matsumura Ind. Pl. Jap. II. 2 (1912) p. 565. Furumi in Tokyo Bot. Mag. XXX. (1916) p. 112.

$M$. nepalensis var. 2 inqua J. D. Hooker hanc reducta huic accedit, attamen foliis majoribus angustioribus, floribus $2-3$ plo longioribus, pedicellis foliis brevioribus ex hac distincta.

Nom. Jap. Mizo-hô-zuki.

Hab.

Yeso: Sapporo prov. Ishikari (KINGo Miyabe). Saruru prov. Hidaka (Y. 'Tokubuchi), sine loco speciali (BoEнmer).

Hondo: Aizu prov. Iwashiro (Jinzō Matsumura). Nikko prov. Shimotsuke (Jinzō Matsumura, Komajirō Sawada). Wadatôge prov. Shinano ((Jinzō Matsumura). Tokujirô prov. Shimotsuke (Saburo Okubo). Sekisuiji prov. Kai (Takenoshin NakaI). Komono prov. Ise (Jinzō Matsumura). Hakusan prov. Kaga (Ryokichi YataBe), prov. Kawachi (T. 'TADA).

Shikoku: Tosa (S. Yano). Kôzusan prov. Awa (Jiurō NikaI).

Kiusiu: Inudake prov. Buzen (R. Yatabe et Jinzo Matsumura). Planta endemica!

481) Rubia cordifolia, IJnné Mantissa (1767) p. 197 Persoon Syn. I. p. 130 p.p. De Candolle Prodr. IV. (1830) p. 588. Ledebouk F1. Ross. II. p. 405.

var. pratensis, Maximowicz Prim. Fl. Amur. p. 140. Regel Tent. Fl. Uss. p. 76. Komarov. Fl. Mansh. III. p. 490. Herder Pl. Radd. III. i. p. 22. Korschinsky in Act. Hort. Petrop. XII. p. 346. NAKaI Fl. Kor. I. p. 294. II. p. 503.

$R$. cordifolia, LINNÉ var. mungista, Y. YABE Fl. Tsus. in Tokyo Bot. Mag. XVIII. (1904) p. 58.

Nom. Jap. Oh-kuruma-akane.

Folia verticillato 6-8 terna (rarius 4 terna) subtus glabra cordatooblonga v. cordato-ovata. Flores $3-4 \mathrm{~mm}$. lati.

Hab.

Corea. Suigen (Номıкі Ueki n. 274). Hangetsujô (Homiki Ueki n. 565). Seoul (Nobutoshi Orada). Namsan (Tomijirô Uchiyama). Makkiri (Takenoshin Nakai n. 5850). Jinsen (Tomijirô Uchiyama). Pyeng-yang (Hanjirô Imai n. 62). Manjyokusan (Takenoshin Nakai). in herbidis Chinnampo (FAurie n. 708).

Manshuria: circa oppidum Tia-o-cho (V. Komarov n. 1442).

Quelpært : sine loco speciali (TAmezô Mori n. 113), monte Hallasan (Takenoshin Nakai n. 970). Hongno (Takenoshin Nakai), insula 
Piyangtô (TAKenoshin NAKAi), in sepibus (TAQUeT n. 5762-3), in herbidis (TAKenoshin NAKaI $n .6477$ ), in sepibus Hongno (TaQuet $n$. 926).

Distr. Amur. Ussuri, Mongolia, Dahuria, Manshuria, Corea. Quelpært et Kiu-siu.

var. silvatica, Maximowicz 1.c. Regel 1.c. Herder 1.c. KorschiNSKY 1.c. KOMAROV 1.c.

R. cordifolia var. hexaphylla, Makino in Tokyo Bot. Mag. XVIII. 1904) p. 144. Matsumura Ind. P1. Jap. II. ii. p. 595.

Folia verticillato 4-8 terna majora quam antea. Flores 4-5 mm. lati.

Hab.

Corea sept.: Hôtaizan (TAkenoshin Nakai).

Hondo : monte Shirouma (Shôsaku Furusawa).

Distr. ut antea et Hondo media.

var. cordata, (Thunberg) NAKaI.

Rubia cordata, Thunberg Fl. Jap. (1784) p. 60. excl. syn.

$R$. Manjith, Roxburgh ex Fleming Catal. in Desvaux Journal de Botanique (1814) part 2. p. 207.

R. Munjista, Roxburgh Fl. Ind. I. (1820) p. 383 . Don Gard. Dict. III. (1834) p. 642. De Candolle Prodr. IV. p. 588. Siebold et Zuccarini Fl. Jap. Fam. Nat. in Abhandl. Muench. Acad. IV. 3. (1846) p. 174.

R. cordifolia, (non LiNì̇) Persoon Syn. I. p. 130 p.p. J.D. Hooker Fl. Brit. Ind. IV. p. 202.

R. cordifolia $\beta$. Mungista, Miquel Prol. Fl. Jap. p. 275. MatsuMURA Ind. Pl. Jap. II. ii. p. 595.

$R$. cordifolia var. mungista, Franchet et Savatier Enum. Pl. Jap. I. p. 212.

R. cordifolia var. laxa, NAKAI Fl. Kor. I. p. 294.

Nom. Jap. Akane.

Folia 4 terna ovata basi cordata apice acuta $v$. acuminata subtus glabra. Flores 3-4 mm. lati.

$\mathrm{Hab}$.

Corea: Namsan (Tamezo Mori). Kongôsan (Tomijiro Uchiyama). Chirisan (TAKenoshin NAKaI).

Quelpært : in sepibus Hongno (TAQUET n. 252).

Hondo: Tokyo (Jinzo Matsumura). Yugashima prov. Idzu (Jinzo Matsumura). Aomori prov. Maitsu (Ryokichi Yatabe). Kônodai prov. Shimousa (Saburô ÔKuBo), insula Ôshima prov. Idzu (Saburô ÔkuBo). Hikami oppidi Ôtuhimura prov. Suwô (Juno NikaI n. 513). 
Yonezawa prov. Uzen (Genichi KoIDzumi).

Shikoku : oppido Kamomyo prov. Awa (JiUrô Nikai n. 2520).

Kiusiu : prov. Buzen (HAMADA).

Distr. Corea: Quelpært, China, Hondo, Shikoku, Kiusiu et India. forma tetramera, MAKINo in Tokyo Bot. Mag. XXI. (1907) p. 251.

Matsumura 1.c.

Flores tetrameri.

Hab. in Hondo, rara!

var. lancifolia, RegeL \{Tent. Fl. Uss. p. 76. Makino in Tokyo Bot. Mag. XVII. (1903) p. 90. Matsumura 1.c. p. 595\} est forma extrema var. pratensis quæ in pratensem sensim transit.

482) Viburnum pubinerye, Blume ms. apud. Miquel Ann. Mus. Bot. Lugd. Bat. II. (1855-6) p. 265.

V. Opulus, (non Linné) Turczaninow Fl. Baic. Dah. I. p. 519. Ledebour Fl. Ross. II. p. 284 p.p. Maximowicz Prim. Fl. Amur. p. 135 et in Mél. Biol. X. p. 670. Ruprecht in Bull. Acad. Petersb. XV. p. 370. Asa Gray Bot. Jap. p. 393. Miquel 1.c. Herder Pl. Radd. III. i. p. 6. BAKER et MOORE in Journ. Linn. Soc. XVII. p. 383. Regel Tent. Fl. Uss. n. 235. Fr. Schmidt Fl. Sachal. n. 218. Forbes et Hemsley in Journ. Linn. Soc. XXIII. p. 354. Palibin Consp. Fl. Kor. I. p. 103. Korshinsky Act. Hort. Petrop. XII. p. 345. Diels in Engler Bot. Jahrb. XXIX. p. 590. Gilg et Loesner in Engler Bot. Jahrb. XXXIV. beiblatt p. 68. NAKAI Fl. Kor. I. p. 286.

V. Sargentii, Koenne Gartenflora XLVIII. (1899) p. 341. SchNEIDER Illus. Handb. Laubholzk II. p. 640, f. 411 a-e. fig. 412. n-q. Rehder Pl. Wils. I. (1911) p. 116.

V. Opulus var. Sargentii, TAKedA in Tokyo Bot. Mag. XXV. p. 25. Nakai Fl. Kur. II. p. 495.

Differt a V. Opulus cortice plus minus suberosa, floribus majoribus, antheris purpureis.

f. puberulum, (Komarov) Nakai.

Vlburnum Sargenti f. puberula, Komarov Fl. Mansh. III. (1907) p. 511.

Folia infra secus venas v. toto pilosa. Pedicelli pilosi v. glabri. Nom. Jap. Kanboku.

Hab.

Sachalin: Perwayaparchi (Genji Nakahara). Kaibatô (Shunzo Komatsu).

Yeso: Konuma prov. Oshima (Ryokichi Yatabe).

Hondo: monte Iwate prov. Rikuchu (Genji Nakahara). Aidzu prov. Iwashiro (Ryokichi Yatabe et Jinzo Matsumura), monte Toga- 
kushi (Jinzo Matsumura), monte Ontake (Jiuro Nikai n. 2215, Genichi KoIdzumi). Nikko prov. Shimotsuke (Komajiro Sawada). Jigami prov. Musashi (Saburo ÓKUBo). Meguro prov. Musashi (Jinzo Matsumura).

Corea: Seishin (Takenoshin Nakai). Mozanrei (Takenoshin Nakai). Paukhan (Sontag). Seoul (Nobutoshi Okada). Chirisan ('Takenoshin Nakai n. 372). Suigen (Homiki Ueki n. 425). Taikori (Takenoshin Nakai n. 2216). Sanyo (Takenoshin Nakai n. 2217). Chanzen (Takenoshin NaKaI n. 5853).

f. calvescens, (REHDER) NAKAI.

V. Sargentii f. calvescens, ReHder in Mitteilung Deut. Dendr. Gesells. XII. (1903) p. 125 et Pl. Wils. I. p. 116. Schneider Illus. Handb. Laubholzk. II. p. 640.

V. Sargentii f. glabra, Komarov Fl. Mansh. III. (1907) p. 511.

Folia subtus glaberrima v. basi venæ tantum pilosæ. Pedicelli et rami glabri.

Nom. Jap. Kenashi-kanboku.

Hab.

Sachalin: Sakaehama (Shunzo Komatsu).

Yeso: sine loco speciali (Boehmer). Muroran (Jinzo Matsumura). Sapporo (Ryokichi YATABE).

Hondo: Okinajima circa Aidzu prov. Iwashiro (Genji Nakahara).

Corea: Onheimen (Takenoshin Nakai n. 7723). Namkanzan (Tamezo Mori n. 24). Taiseizan (Hanjiro Imai n. 32). Hakuhekizan (Tsutomu Ishidoya). Seoul (Nobutoshi Okada). Kôryo (Tamezo Mori n. 199). Kôshu (Takenoshin Nakai n. 1024). Kôkai (TakeNoshin Nakai n. 2257), Sakushu (Takenoshin NaKai n. 2249). Kongosan (Takenoshin Nakai n. 5852).

Manshuria : circa stationem Radde (V. Komarov n. 1450).

f. Iutescens, NakaI.

V. Opulus f. sterile, (non Dippel) Palibin Consp. Fl. Kor. I. p. 103. NaKaI Fl. Kor. I. p. 286.

V. Opulus var. Sargentii f. sterile, NAKAI Fl. Kor. II. p. 495.

Ut forma antea sed flores omnes steriles. Corolla lutescens.

Nom. Jap. Kanboku-Azisai.

Hab.

Corea: in horto Suigen (Homiki UekI, Takenoshin Nakai), in monte Yisan (SonTaG).

var. hydrangeoides, NAKaI.

Foliorum forma ut antea sed duplo v. triplo majora. Flores albi omnes steriles 10-18 $\mathrm{mm}$. lati. Inflorescentia maxima ambitu subsphærica. 
Nom. Jap. Temari-kamboku.

$\mathrm{Hab}$.

Corea: in horto templi Kôryo (TAEnoshin NAKaI n. 2226). 483) Patrinia gibbiferum, NakaI sp. nov.

P. palmata var. gibbosa, MAKINo in Tokyo Bot. Mag. XXI. (1907) p. 157.

Differt a $P$. palmato caule elatiore et robustiore foliis majoribus, floribus duplo minoribus et basi non calcaratis sed tantum gibbosis.

Nom. Jap. Ko-kinreikwa.

$\mathrm{Hab}$.

Hondo: lidesan prov. Iwashiro (Genj Nakahara). Nikko (Saburō Ōkubo). Asamayama prov. Shinano (Jinzō Matsumura). Togakushi prov. Shinano (Jinzō Matsumura). Ontake prov. Shinano (Saburō Ókubo). Yatsugatake (Yoshitada Yabe). Hakusan prov. Kaga (Jinzō Matsumura).

484) Aster incisus, Fischer in Memoires Societ. Imperiale Naturalistes de Moscou III. (1812) p. 76. Forbes et Hemsley in Journ. Linn. Soc. Bot. XXIII. p. 412. Palib. Consp. Fl. Kor. I. p. 110. Diels in Engl. Bot. Jahrb. XXIX. p. 610. Komarov Fl. Mansh. III. p. 598. Nakai Fl. Kor. II. p. 7.

Calimeris incisa, De Candolle Prodr. V. (1836) p. 258. Turczaninow Fl. Baic.-Dah. II. p. 17. Ledebour Fl. Ross. II. p. 482. Maximowicz Prim. Fl. Amur. p. 145. Regel Tent. Fl. Uss. p. 83. 11. 255. Fr. Schmidt Fl. Amg. n 204. Herder Pl. Radd. III. ii. p. 17. Korschinsky in Act. Hort. Petrop. XII. p. 350.

Boltonia incisa, Bentham Fl. Hongk. (1861) p. 175. Miquel Prol. Fl. Jap. p. 102. Franchet et Savatier Enum. Pl. Jap. I. p. 225.

Heteropappus incisus, Siebold et Zuccarini Fl. Jap. Fam. Nat. in Abhandl. Akad. Muench. IV. 3. (1846) p. 182.

Calimeris platycephala, NeEs Synops. Sp. Gen. Aster. (1818) p. 227.

Grindelia incisa, Sprengel Syst. Veg. III. (1826) p. 575.

Kalimeris platycephala, CAssinI Dict. XXIV. (1826) p. 325.

Aster indicus, (non Linné) ThunbFrg Fl. Jap. (1784) p. 316. Nakai Fl. Kor. II. p. 8. Makino Somokudzusetsu Rev. ed. IV. (1912) p. 32. P1. 38.

Boltonia indica, (non Bentham) Mrgtel Prol. Fl. Jap. p. 101. Franchet et Savatier Enum. Pl. Jap. I. p. 225 excl. syn.

Asteromæa indica, (non Blume) Asa Gray Perry's Exp. (1857) p. 314. YaBe Fl. Tsus. in Tokyo Bot. Mag. XVIII. (1904) p. 60. 
Matsumura et Koldzumi in Tokyo Bot. Mag. XXIV. p. 94.

Nom. Jap. Yomena.

Nom. Corea: Supuzien.

Hab. Davuria, Manshuria, Corea, Quelpaert, Hondo, Shikoku, insula Tsusima et Kiusiu.

Hondo: Aomori prov. Mutsu (Ryokichi Yatabe, Nobutaro Kinashi), inter Hagurosan et Tsurugaoka prov. Uzen (Saburo Okubo), Nikko prov. Shimotsuke (Jinzo Matsumura, Komajiro Sawada). Shidagori prov. Hitachi (Jinzo Matsumura), Ontakesan prov. Shinono (GeNichi Koidzumi). Wakimachi prov. Awa (Saburo Okubo). Tokyo (Saburo Okubo, Komajiro Sawada). Asakumayama prov. Ise (TakeNoshin NAKAI). Kawachi ( $T$. TADA). Hikami prov. Suwo (JIURo NikaI n. 589).

Shikoku: Kamomyomura prov. Awa (JiUro NikaI n. 2534).

Insula Tsusima: Takeshiki (Yoshitada YaBE).

Kiusiu: Buzen (Hamada) Ureshino prov. Hizen (Jinzo Matsu MLRA).

Corea: Fluvium Jalu superior. Vallis Karami (V. Komarov n. 1507), inter Keizanchin et Futempo (TAKENoshin NakaI n. 2773). inter Shozando et Hoangguito (TAKENOSHIN NAKAI n. 2785). Sakjyu (Mills 11. 632). Kangei (Mills n. 740, 4.10, 108, 187, 192, 145, 141, 48, 136). Zuiko (TAkenoshin Nakai n. 2878). Seoul (NobUtoshi (Kada). Hokkanzan (Tomiriro Uchiyama). Namsan (NobUtoshi Okada, Faurie n. 1071, 393). Fusan (Motogoro Enuma).

Quelpært: in herbidis (Takenoshin NakaI n. 6519). Hongno (TaQuet n. 951). Hallasan (TaqueT n. 950).

var. holophylla, Maximowicz apud Komarov Fl. Mansh. III. p. 599.

Folia integra rarius paucidentata.

Hab.

Corea: Fusan (Faurie n. 1051 p.p.). Hakuhekisan (Tsutomu Ishidoya). Sensen (Mills n. 979).

Manshuria: prov. Kirinensis (V. Komarov n. $1507 \beta$ ).

var. pinnatifidus, (Matsumura) NakaI.

Boltonia indica, Linné var. pinnatifida, Matsumura Catal. Herb. Coll. Sci. Imp. Univ. Tokyo (1886) Correct. p. 3.

Asteromæa indica var. pinnatifida, Matsumura Shokubutsumeii (1903) p. 41 et Ind. Pl. Jap. II. 2 (1912) p. 629. Matsumura et Koidzumi in Tokyo Bot. Mag. XXIV. p. 94.

Aster indicus, LinNé var. pinnatifida, Maximowicz in litt. apud Makino in Tokyo Bot. Mag. XX. (1906) p. 41. 
Aster pinnatifidus, Makino Somokudzusetsu Rev. ed. IV. p. 40. Pl. L.

Nom. Jap. Yügagiku.

Hab. Corea, Quelpært et Hondo.

Corea: Shōshuku (Nobutoshi Okada). Chanzen (Takenoshin Nakai n. 5969). Suigen (Homiki Ueki n. 232, 217). Kyojyo (Tamezo MORI n. 282).

Quelpært: in herbidis (TAKenoshin NAKaI n. 6522). Hoatien (TAQuet n. 6236). Hallasan (TAQuet n. 226 p.p.).

Hondo: Horinouchi prov. Musashi (Saburo Okubo, Jinzo Matsumura). Nikko prov. Shimotsuke (Jinzo Matsumura). Nobitome (Saburo Okubo). Aidzu prov. Iwashiro (Jinzo Matsumura). Shidagôri prov. Hitachi (Jinzo Matsumura). Ohmineyama prov. Shinano (Jinzo Matsumura).

485) Aster indicus, Linné Sp. Pl. (1753) p. 876 et Syst. Veg. ed. 14 (1784) p. 762. Houttuyn Pflanzensyst. IX. (1783) p. 387. WILLDenow Sp. Pl. III. p. 2041. Dietrig Garten Lexicon I. (1802) p. 13. Persoon Syn. Pl. II. 2. (1807) p. 44. Hayata Composit. Form. p. 14 et Ind. Pl. Form. p. 37. Matsumura et Hayata Enum. Pl. Form. p. 204.

Asteromaea indiea, Blume Bijidragen (1825) p. 901. De Candolle Prodr. V. (1836) p. 303. Mrquet. Fl. Nederland. Indie II. (1856) p. 29. Callistemma indicum, G. Dow in Loudon Hort. Brit. t. 348.

Boltonia indica, Bentham Fl. Hongk. (1861) p. 174. Hooker fil. Fl. Brit. Ind. III. (1881) p. 249 (excl. Calimeris integrifolia).

Chrysanthemum cuneatum, RoxBURGH Fl. Ind. III. p. 436.

Matricaria cantoniensis, LoureIro apud Willdenow Sp. Pl. III. p. 2041.

Hisutsua cantoniensis, De Candolle Prodr. VI. (1837) p. 44.

Aster ursinus, Léveillé in Fedde Rep. XII. (1913) p. 100.

Nom. Jap. Indo-yomena.

Nom. Quelp. Truk-kwa.

Hab. Corea austr., Quelpært, Bonin, Formosa, China, India, Cochin-china, Burma, Malaya et Java.

Corea: Nangen (TAmezō Mori n. 335).

Quelpært: in humidis (TAQUET n. 5675), in herbidis (TAKENOSHIN Nakai n. 6520, Tsutomu Ishidoya n. 157).

Bonin : in insula Chichisima (Shigetsugu Nishimura).

Liukiu: in insula Okinawa (Tetsuo Mryagi), in insula Yaeyama (Yasusada Tashiro), in Shuri insulæ Okinawa (KIICHI MiYake).

Formosa: Taihoku (Tomitarō Makino). Kelung (Tomitarō Makino). Shabosansho (?), Tamsui (S. Nagasawa). 